\title{
Circulating Levels of VEGF and CXCL1 Are Predictive of Metastatic Organotropismin in Patients with Colorectal Cancer
}

\author{
ROSA DIVELLA $^{1}$, ANTONELLA DANIELE ${ }^{1}$, RAFFAELE DE LUCA ${ }^{2}$, MICHELE SIMONE ${ }^{2}$, EMANUELE \\ NAGLIERI $^{3}$, EUFEMIA SAVINO ${ }^{1}$, INES ABBATE ${ }^{1}$, COSMO DAMIANO GADALETA $^{4}$ and GIROLAMO RANIERI ${ }^{4}$ \\ ${ }^{1}$ Clinical Pathology Laboratory, ${ }^{2}$ Department of Surgery Oncology, ${ }^{3}$ Department of Medical Oncology, and \\ ${ }^{4}$ Diagnostic and Interventional Radiology Unit with Integrated Section of Translational Medical Oncology, \\ National Cancer Research Centre, Giovanni Paolo II Tumor institute, Bari, Italy
}

\begin{abstract}
Colorectal cancer is the most common cancer of the gastrointestinal system and has a marked preference to metastasize to distant organs. In this study, we investigated whether levels of circulating serum pro-angiogenic cytokine such as chemokine ( $\mathrm{C}-\mathrm{X}-\mathrm{C}$ motif) ligand 1 (melanoma growth-stimulating activity, alpha; CXCL1) and vascular endothelial growth factor (VEGF) have a role in favoring the colonization of metastatic cells at preferential sites and determined their prognostic significance in a cohort of 103 patients with metastatic colorectal cancer. Importantly, we found that the presence of elevated circulating levels of VEGF and CXCL1 are predictive of liver and lung metastasis, respectively. Moreover, the presence of a high serum VEGF level represents a negative prognostic factor for patients with liver metastases, with a worse prognosis than patients with lung metastasis. This suggests an additional role for circulating cytokines as a predictive tool for cancer prognosis and diagnosis, as well as for assessment of tumor sensitivity to anticancer therapy.
\end{abstract}

Similarly to other types of tumor, colon cancer also metastases to preferential organs. More than $70 \%$ of patients with colorectal cancer have liver metastases. The lung is the second most important organ for colorectal carcinoma metastasis, and 20 to $30 \%$ of all distant colorectal carcinoma metastases are found primarily in the lung $(1,2)$. According to 'the seed and soil theory', postulated by Stephen Paget in 1889, there is a propensity for certain tumors to seed to particular organs (3, 4). A tumor cell becomes metastatic by establishing interactions with homeostatic factors in a target organ, thus

Correspondence to: Rosa Divella, Department of Experimantal Oncology, Viale Orazio Flacco, 65, 70100, Bari, Italy. Tel: +39 0805555259, e-mail: rosadive@inwind.it

Key Words: Metastasis, angiogenesis, CXCL1, VEGF. promoting cell survival, angiogenesis and tumor growth. These steps are mediated by specific adhesive interactions between cancer cells and the host organ microvasculature $(5$, 6 ). Recent studies have demonstrated the presence in the target organs of specific molecules that include chemokines, growth factors and adhesion molecules that interact with receptors or ligands present on tumor cells that together contribute to the formation of organ-specific metastasis (7-9).

The formation of new blood vessels is determined by the secretion of pro angiogenic factors by tumor cells. Vascular endothelial growth factor (VEGF) is the most important modulator of tumor angiogenesis and performs important activities including reducing the functions of the biological barrier and regulating endothelial sprouting $(10,11)$. It also increases vascular permeability and, through VEGF-vascular endothelial growth factor receptor (VEGFR) interactions, allows the mobilization and recruitment of endothelial cells within tumors. Many published studies show that VEGF plays an important role in the development of colon cancer, especially in the stimulation of angiogenesis $(12,13)$. Given that VEGF is expressed in various types of tumors, determination of circulating levels of VEGF could provide a diagnostic method of great use in patients with cancer (14). An increase in VEGF expression in tumour tissue or blood compartments (i.e. serum or plasma) has been found in solid and hematological malignancies of various origins and is associated with metastasis formation and poor prognosis (15, 16). By multivariate analysis of molecular markers for detection of liver metastases after surgery in patients with colorectal cancer, it was demonstrated that a combination of markers such as carcinoembryonic antigen (CEA), epidermal growth factor receptor (EGFR) and VEGF could be an effective method for predicting liver metastases in patients with colorectal cancer (17).

Chemokines such as chemokine (C-X-C motif) ligand 1 (melanoma growth-stimulating activity, alpha; CXCL1) are intricately associated with cellular transformation, tumor growth and increase of invasive potential $(18,19)$. Many 
chemokines, including CXCL1, are strong inducers of chemotaxis and there are several lines of evidence showing that they play a role in tumor progression, for example, by increasing metastasis formation in preferential target organs (20-23). In this study, we investigated whether levels of circulating serum cytokine such as CXCL1 and VEGF have a role in favoring the colonization of metastatic cells to preferential sites and determined their prognostic significance in patients with metastatic colorectal cancer.

\section{Patients and Methods}

Patients and blood samples. The group of patients examined in this study is the same as reported in a previous work (24). In short, in this analysis, 103 patients, 65 male and 38 female, with median age 65 (range $=39-82$ ) years who underwent curative hepatic and pulmonary metastases from colorectal cancers treatable with resection or tumor ablation were enrolled in this prospective study at the Giovanni Paolo II National Cancer Institute, Bari Italy. Patients with synchronous liver or lung metastases were included if the resection of metastases was performed together with that of the primary tumor.

A total of 69 patients $(67 \%)$ had liver metastases, while $34(33 \%)$ had lung metastases; 30 patients $(29.1 \%)$ had synchronous metastasis, while 73 patients $(70.9 \%)$ had metachronous metastasis. Only five patients $(4.8 \%)$ had synchronous presentation of liver or lung metastasis. A total of 47 patients $(45.6 \%)$ had solitary metastasis and 58 patients $(56.3 \%)$ had unilobular distribution of metastasis. The clinical characteristics of patients (age, sex, therapeutic interventions, etc.) were obtained from medical records. In accordance with national and institutional standard procedures, all patients received systemic therapy: 35 patients underwent neoadjuvant chemotherapy, and 68 patients received neoadjuvant bevacizumab treatment. Blood samples were taken on the day of diagnosis from newly diagnosed patients with synchronous metastasis or from patients who developed metastases metachronously after R0-resection of the primary tumor. For analysis of cytokines, $5 \mathrm{ml}$ of peripheral blood were collected in a vacutainer system and serum was immediately separated from the cellular fraction by centrifugation at $1,500 \times g$ for $10 \mathrm{~min}$ and frozen at $-20^{\circ} \mathrm{C}$. Blood samples from 50 healthy individuals were used as negative controls. Written consent was obtained from all patients prior to enrolment in the study and the Ethical Committee of the Institute approved the protocol in accordance with the ethical guidelines of the 1975 Declaration of Helsinki.

CXCL1 and VEGF enzyme-linked immunoassay (ELISA). Serum samples from patients and healthy donors were assayed for levels of CXCL1 and VEGF by a sandwich ELISA assay (Quantikine Human CXCL1 and VEGF Immunoassay; R\&D Systems, Inc., Minneapolis, USA) according to the manufacturer's recommendations. The absorbance of the solution produced was measured at $490 \mathrm{~nm}$ and was directly proportional to the amount of CXCL1 and VEGF present in the sample. A standard curve was constructed by plotting the mean absorbance value measured for each standard versus its corresponding concentration. The minimal detection limit was $10 \mathrm{pg} / \mathrm{ml}$ for CXCL and $1.5 \mathrm{pg} / \mathrm{ml}$ for VEGF.

Statistical analysis. The association between serum levels of CXCL1 and VEGF with clinical characteristic of patients was analyzed with unpaired $t$-test and ANOVA. Spearman's correlation
Table I. Correlation between circulating cytokines and clinicopathological variables in patients with metastatic colorectal cancer.

\begin{tabular}{lccccc}
\hline & N $(\%)$ & $\begin{array}{c}\text { VEGF } \\
(\mathrm{pg} / \mathrm{ml})\end{array}$ & $p$-Value* & $\begin{array}{c}\text { CXCL1 } \\
(\mathrm{pg} / \mathrm{ml})\end{array}$ & $p$-Value* \\
\hline $\begin{array}{l}\text { Control group } \\
\text { Patients }\end{array}$ & 50 & $88 \pm 14$ & $<0.0001$ & $60 \pm 12$ & $<0.0001$ \\
Gender & 103 & $500 \pm 391$ & & $410 \pm 146$ & \\
$\quad$ Female & $38(36.9)$ & $646 \pm 520$ & & $411 \pm 126$ & \\
$\quad$ Male & $65(63.1)$ & $365 \pm 108$ & & $409 \pm 167$ & \\
Metatastic site & & & 0.004 & & 0.001 \\
$\quad$ Liver & $69(66.9)$ & $453 \pm 156$ & & $325 \pm 123$ & \\
$\quad$ Lung & $34(33.1)$ & $354 \pm 163$ & & $415 \pm 136$ & \\
Time of metastasis & & & $<0.0001$ & & 0.008 \\
$\quad$ Synchronous & $30(29.1)$ & $458 \pm 256$ & & $325 \pm 205$ & \\
$\quad$ Metachronous & $73(70.9)$ & $254 \pm 200$ & & $423 \pm 150$ & \\
No. of metastases & & & $<0.0001$ & & $<0.0001$ \\
$\quad 1$ & $47(45.6)$ & $349 \pm 230$ & & $235 \pm 158$ & \\
$\quad$ >1 & $56(54.3)$ & $658 \pm 260$ & & $452 \pm 122$ & \\
Neoadjuvant therapy & & & $<0.0001$ & & n.s. \\
$\quad$ Yes & $35(33.9)$ & $258 \pm 125$ & & $430 \pm 123$ & \\
$\quad$ No & $68(66.1)$ & $542 \pm 200$ & & $400 \pm 136$ & \\
Neoadjuvant & & & & & \\
bevacizumab & & & $<0.0001$ & & n.s. \\
$\quad$ Yes & $20(19.4)$ & $260 \pm 165$ & & $410 \pm 123$ & \\
$\quad$ No & $83(80.6)$ & $600 \pm 130$ & & $409 \pm 167$ & \\
\hline
\end{tabular}

VEGF: Vascular endothelial growth factor; CXCL1: chemokine (C-XC motif) ligand 1 (melanoma growth stimulating activity, alpha); n.s.: not significant. *By ANOVA and $t$-test.

was used for the correlation analysis, and the survival analysis was evaluated with Kaplan-Meier and log-rank test. Overall survival (OS) was defined as the time between the date of the blood sample draw and the date of death or the last follow-up examination. A $p$ value of 0.05 or less was taken to indicate statistical significance. All statistical analyzes were performed by Number Cruncher Statistical System-Power Analysis and Sample Size Software 2007 NCSS-PASS, (Statistical Analysis and sample Size - Power Analysis and Graphics, Kaysville, UT, USA).

\section{Results}

Association between circulating levels of cytokines and clinicopathological parameters. The serum levels (mean \pm SD) of CXCL1 and VEGF are reported in Table I. The mean values of these cytokines were much higher in patients than in healthy donors ( $p=0.0001$, ANOVA). A significant association between female gender and circulating levels of VEGF was found $(p<0.0001)$. Considering serum levels of the two cytokines according to metastatic site, we interestingly noted that higher serum levels of VEGF were associated with the presence of liver metastases $(p=0.004)$, while higher levels of CXCL1 were associated with the presence of lung metastases $(p=0.001)$. Lastly, levels of CXCL1, in contrast to VEGF, were inversely associated with 


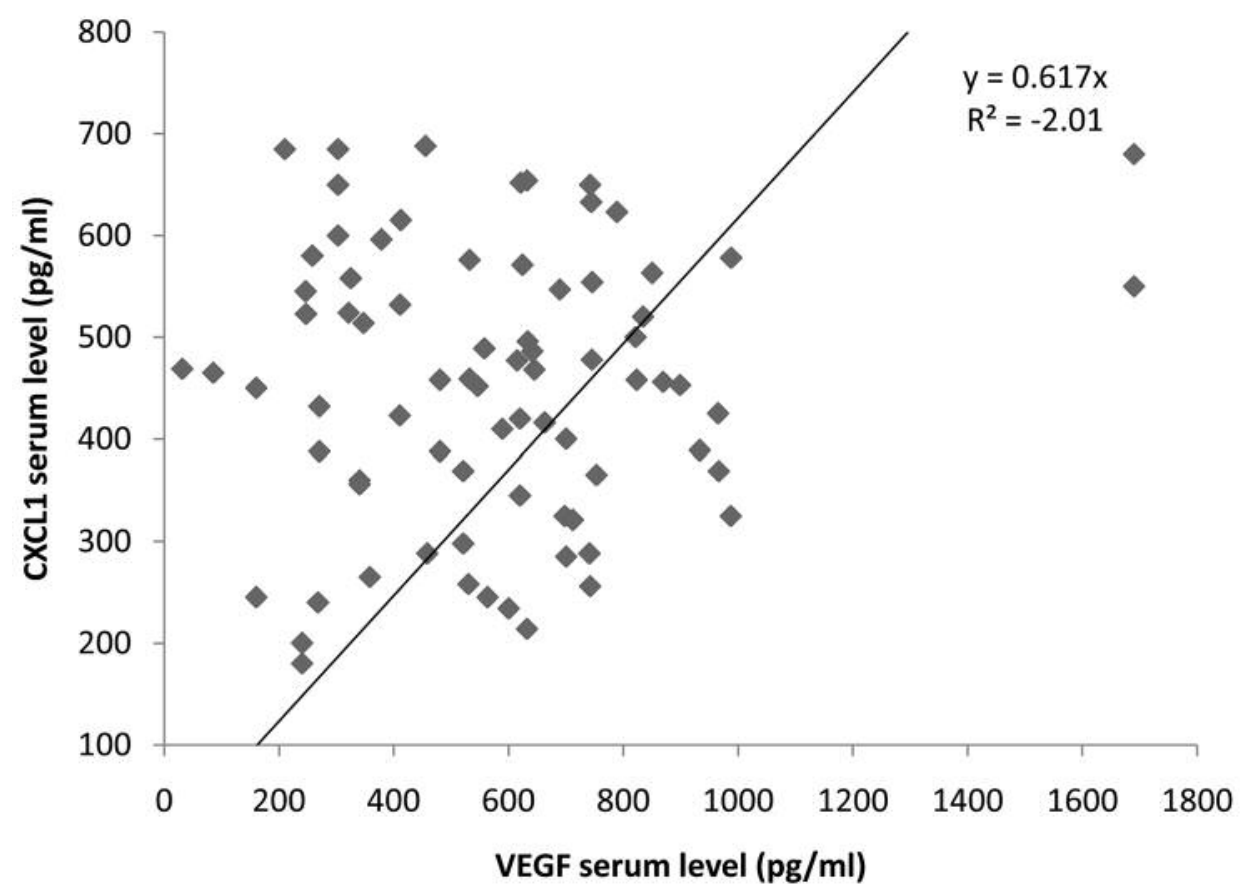

Figure 1. Scatter plot showing the correlation between serum levels of vascular endothelial growth factor (VEGF) and chemokine (C-X-C motif) ligand 1 (CXCL1). Spearman correlation, $p<0.0001$.

synchronous metastases $(p=0.008$ for CXCL1 and $p<0.0001$ for VEGF).Therefore, we evaluated the correlation between these cytokines. As shown in Figure 1, in our analysis, we found significant positive correlation between CXCL1 and VEGF (Spearman correlation, $p<0.0001$ ).

Higher serum VEGF is associated with worse prognosis in patients with liver metastasis. For patients overall, the median OS was 24 months as shown in Table II. Multiple variables such as gender, time of metastasis and metastatic site affecting survival were examined by univariate analysis using the log-rank test. Univariate log-rank analysis showed that shorter survival was observed in males than in females (20 vs. 26 months, respectively, $p<0.004)$. Moreover, patients with metachronous metastases had a poorer prognosis compared with patients with synchronous metastasis, with median OS of 20 months versus 25 months $(p<0.003)$. Finally, a shorter survival was associated with hepatic metastasis, with a median OS of 17 versus 24 months $(p=0.02)$ for patients with pulmonary metastasis.

We, therefore, established a cutoff value for CXCL1 $(410 \mathrm{pg} / \mathrm{ml})$ and VEGF $(500 \mathrm{pg} / \mathrm{ml})$ by receiver operating curve analysis. Considering survival according to the two cytokines, patients with VEGF higher than the cutoff value of $\geq 500 \mathrm{pg} / \mathrm{ml}$ had a worse prognosis than patients with
Table II. Mean (range) overall survival (OS) in correlation to gender, time of metastasis, metastatic site and serum vascular endothelial growth factor (VEGF) and chemokine (C-X-C motif) ligand 1 (CXCL1) levels.

\begin{tabular}{llc}
\hline & OS (months) & $p$-Value* \\
\hline All patients (103) & $24(3-32)$ & \\
Female & $26(5-32)$ & $<0.004$ \\
Male & $20(3-27)$ & \\
Synchronous metastasis & $20(3-26)$ & $<0.003$ \\
Metachronous metastasis & $25(8-32)$ & \\
Liver metastasis & $18(3-22)$ & $<0.0001$ \\
Lung metastasis & $26(7-32)$ & \\
VEGF & & \\
$\quad \geq 500 \mathrm{pg} / \mathrm{ml}$ & $17(3-20)$ & $<0.0001$ \\
$\quad<500 \mathrm{pg} / \mathrm{ml}$ & $25(4-25)$ & \\
CXCL1 & & \\
$\geq 410 \mathrm{pg} / \mathrm{ml}$ & $22(3-25)$ & $<0.005$ \\
$<410 \mathrm{pg} / \mathrm{ml}$ & $26(8-32)$ & \\
\hline
\end{tabular}

*By Kaplan-Meier and log-rank test.

lower values (17 vs. 25 months, $p<0.0001)$. Furthermore, patients with values of CXCL1 higher than the cutoff of $\geq 410 \mathrm{pg} / \mathrm{ml}$ had a worse prognosis than patients with lower values (22 vs. 26 months, $p=0.02$ ). 


\section{Discussion}

Metastases are the result of a complex cascade of events that begins with the detachment of tumor cells from the primary site and their reaching distant organs where they proliferate and form secondary tumor foci. During their stay in the bloodstream, tumor cells undergo a series of events that are regulated by an interaction of molecules secreted by both tumor cells and host cells and that will define the phenotypic fate of tumor cells (25). The formation of microvessels around the tumor is mediated by the presence of various angiogenic growth factors secreted by tumor cells (23). VEGF is one of the most powerful endothelial growth factors and acts by stimulating mitogenesis of endothelial cells; it is also considered one of the strongest factors promoting angiogenesis in colorectal cancer (26). In patients with colorectal cancer, VEGF levels are enhanced and provide an index of disease spread and prognosis $(27,28)$. Several studies have shown that angiogenesis is a negative prognostic factor for both overall and disease-free survival in patients with colorectal cancer (29). Most malignant tumors display an organ-specific metastatic pattern. For example, colon cancer cells preferentially colonize the lungs and liver, where they will develop metastases (30, 31). In other organs such as bone or brain, it is rare to observe the presence of colorectal cancer metastasis.

In this study, we have shown that the presence of higher circulating levels of pro-angiogenic cytokines VEGF and CXCL1 may be useful predictors of occurrence of liver and lung metastases, respectively, in patients with colorectal cancer, confirming that colon cancer cells preferentially target the liver and the lung to establish secondary tumors. We have also shown that VEGF is a biomarker of adverse prognosis in those patients who developed liver metastases. In fact, our study has shown that the presence of a high serum VEGF level in patients with liver metastasis was associated with reduced survival rate compared to those who developed pulmonary metastases. From these data, we can hypothesize that during their time in the circulation, cancer cells undergo various transformations taking on various phenotypes that predispose them to colonizing certain organs rather than others. In this case, high circulating levels of VEGF in patients with liver metastases suggests that the liver is an organ where metastatic cells appear to be phenotypically more aggressive than those that have colonized the lungs.

Organoselectivity is mediated by molecular factors such as chemokines and cytokines that are released into the microenvironment both from host cells and cancer cells (32, 33). In fact, it is thought that it is precisely the interaction between the cytokine receptors present on tumor cells and the ligands of the target organs which modulates the organotropism of metastasis. Constitutive expression of CXCL1 and its receptor CXCR2 was reported to regulate cell proliferation and invasive phenotype in colorectal cancer cell lines (34). In a study by Massague et al. that compared the genetic pattern of breast cancer cell lines with weak or strong metastatic tropism for the lung, CXCL1 was certainly one of the genetic products that promoted pulmonary metastasis (35). In our study, we confirmed that the expression of chemokine CXCL1 facilitates cell seeding and outgrowth of metastases at distant sites. CXCL1 also plays an important role in the recruitment of cancer cells to the lung in patients with metastatic colon cancer (36). This could open up new treatment opportunities to destroy tumor metastases by creating drugs that target chemokines or their receptors. Although CXCL1 promotes tumor cell growth and is involved in angiogenesis of several cancer types in animal models, its role in human cancer needs to be clarified. Although correlations can be found between the primary tumors and their preferential metastatic sites, there is not yet a rationale underlying organ preference.

A genetic fingerprint indicating which genes play a significant role in the metastasis of primary tumors preferentially to specific organs has been identified (37). Deeper knowledge about the mechanisms that govern the link between genes and the fate of metastatic cells could present an opportunity for improved patient care and for the prevention of many deaths from cancer.

\section{Conflicts of Interest}

The Authors declared that they have no competing interests with regard to this study.

\section{References}

1 Robinson JR, Newcomb PA, Hardikar S, Cohen SA and Phipps AI: Stage IV colorectal cancer primary site and patterns of distant metastasis. Cancer Epidemiol 48: 92-95, 2017.

2 Kim JY, Park IJ, Kim HR, Kim DK, Lee JL, Yoon YS, Kim CW, Lim SB, Lee JB, Yu CS and Kim JC: Post-pulmonary metastasectomy prognosis after curative resection for colorectal cancer. Oncotarget, 2017. doi: 10.18632/oncotarget.16616. [Epub ahead of print]

3 Paget S: The distribution of secondary growth in cancer of the breast. Lancet 1: 571-573, 1889.

4 Fidler IJ: The pathogenesis of cancer metastasis: the 'seed and soil' hypothesis revisited. Nat Rev Cancer 3: 453-458, 2003.

5 Balzer EM and Konstantopoulos K: Intercellular adhesion: mechanisms for growth and metastasis of epithelial cancers. Wiley Interdiscip Rev Syst Biol Med 4(2): 171-181, 2012.

6 Brooks SA, Lomax-Browne HJ, Carter TM, Kinch CE and Hall DM: Molecular interactions in cancer cell metastasis. Acta Histochem 112(1): 3-25, 2010.

7 Richmond A: Chemokine modulation of the tumor microenvironment. Pigment Cell Melanoma Res 23(3): 312-313, 2010.

8 Spano D, Heckc C, De Antonellis P, Christofori G and Zollo M: Molecular networks that regulate cancer metastasis. Semi Cancer Biol 22: 234-249, 2012.

9 Balkwill F: Cancer and the chemokine network. Nat Rev Cancer 4: 540-550, 2004. 
10 Auyeung KK and Ko JK: Angiogenesis and oxidative stress in metastatic tumor progression: pathogenesis and novel therapeutic approach of colon cancer. Curr Pharm Des, 2017. doi: 10.2174/1381612823666170228124105. [Epub ahead of print]

11 Bendardaf R, El-Serafi A, Syrjänen K, Collan Y and Pyrhönen $\mathrm{S}$ : The effect of vascular endothelial growth factor-1 expression on survival of advanced colorectal cancer patients. Libyan J Med 12(1): 1290741, 2017.

12 Canavese M, Ngo DT, Maddern GJ, Hardingham JE, Price TJ and Hauben E: Biology and therapeutic implications of VEGFA splice isoforms and single-nucleotide polymorphisms in colorectal cancer. Int J Cancer 140(10): 2183-2191, 2017.

13 Marech I, Leporini C, Ammendola M, Porcelli M, Gadaleta CD, Russo E, De Sarro G and Ranieri G: Classical and non-classical proangiogenic factors as a target of antiangiogenic therapy in tumor microenvironment. Cancer Lett 380(1): 216-226, 2016.

14 Di Caro G, Carvello M, Pesce S, Erreni M, Marchesi F, Todoric J, Sacchi M, Montorsi M, Allavena P and Spinelli A: Circulating inflammatory mediators as potential prognostic markers of human colorectal cancer. PLoS One 11(2): e0148186, 2016.

15 Mehrabani D, Shamsdin SA, Dehghan A and Safarpour A: Clinical significance of serum vascular endothelial growth factor and complement 3a levels in patients with colorectal cancer in southern Iran. Asian Pac J Cancer Prev 15(22): 9713-9717, 2014.

16 Beştaş R, Kaplan MA, Ișikdoğan A: The correlation between serum VEGF levels and known prognostic risk factors in colorectal carcinoma. Hepatogastroenterology 61(130): 267-271, 2014.

17 Qian LY, Li P, Li XR, Chen DJ and Zhu SH: Multivariate analysis of molecular indicators for postoperative liver metastasis in colorectal cancer cases. Asian Pac J Cancer Prev 13(8): 3967-3971, 2012.

18 Wani N, Nasser MW, Ahirwar DK, Zhao H, Miao Z, Shilo K and Ganju RK: C-X-C motif chemokine 12/C-X-C chemokine receptor type 7 signaling regulates breast cancer growth and metastasis by modulating the tumor microenvironment. Breast Cancer Res 16(3): R54, 2014.

19 Ogata H, Sekikawa A, Yamagishi H, Ichikawa K, Tomita S, Imura J, Ito Y, Fujita M, Tsubaki M, Kato H, Fujimori T and Fukui H: GROa promotes invasion of colorectal cancer cells Oncol Rep 24(6): 1479-1486, 2010.

20 Divella R, Daniele A, Savino E, Palma F, Bellizzi A, Giotta F, Simone G, Lioce M, Quaranta M, Paradiso A and Mazzocca A: Circulating levels of transforming growth factor- $\beta$ eta (TGF- $\beta$ ) and chemokine (C-X-C motif)ligand-1 (CXCL1) as predictors of distant seeding of circulating tumor cells in patients with metastatic breast cancer. Anticancer Res 33(4): 1491-1497, 2013

21 Spaks A: Role of CXC group chemokines in lung cancer development and progression. J Thorac Dis 9(Suppl 3): S164S171, 2017.

22 Han KQ, Han H, He XQ, Wang L, Guo XD, Zhang XM, Chen J, Zhu QG, Nian H, Zhai XF and Jiang MW: Chemokine CXCL1 may serve as a potential molecular target for hepatocellular carcinoma. Cancer Med 5(10): 2861-2871, 2016.

23 Miyake M, Hori S, Morizawa Y, Tatsumi Y, Nakai Y, Anai S, Torimoto K, Aoki K, Tanaka N, Shimada K, Konishi N, Toritsuka M, Kishimoto T, Rosser CJ and Fujimoto K: CXCL1-Mediated interaction of cancer cells with tumor-associated macrophages and cancer-associated fibroblasts promotes tumor progression in human bladder cancer. Neoplasia 18(10): 636-646, 2016.
24 Divella R, Daniele A, Abbate I, Bellizzi A, Savino E, Simone G, Giannone G, Giuliani F, Fazio V, Gadaleta-Caldarola G, Gadaleta CD, Lolli I, Sabbà C and Mazzocca A: The presence of clustered circulating tumor cells (CTCs) and circulating cytokines define an aggressive phenotype in metastatic colorectal cancer. Cancer Causes Control 25(11): 1531-1541, 2014.

25 Cuyle PJ and Prenen H: Current and future biomarkers in the treatment of colorectal cancer. Acta Clin Belg 72(2): 103-115, 2017.

26 Tirumani SH, Fairchild A, Krajewski KM, Nishino M, Howard SA, Baheti AD, Rosenthal MH, Jagannathan JP, Shinagare AB and Ramaiya NH: Anti-VEGF molecular targeted therapies in common solid malignancies: comprehensive update for radiologists. Radiographics 35(2): 455-474, 2015.

27 Liu W, Xu J, Wang M, Wang Q, Bi Y and Han M: Tumor-derived vascular endothelial growth factor (VEGF-A) facilitates tumor metastasis through the VEGF-VEGFR1 signaling pathway: Int $\mathrm{J}$ Oncol 39: 1213-1220, 2011.

28 Suenaga M, Mashima T, Kawata N, Wakatsuki T, Horiike Y, Matsusaka S, Dan S,Shinozaki E, Seimiya H, Mizunuma N, Yamaguchi K and Yamaguchi T: Serum VEGF-A and CCL5 levels as candidate biomarkers for efficacy and toxicity of regorafenib in patients with metastatic colorectal cancer. Oncotarget 7(23): 34811-34823, 2016.

$29 \mathrm{Xu} \mathrm{B}$, Shen F, Cao J and Jia L: Angiogenesis in liver metastasis of colorectal carcinoma. Front Biosci (Landmark Ed) 18: 1435-1443, 2013.

30 Schluter K, Gassmann P, Enns A, Korb T, Hemping-Bovenkerk A, Holzen $\mathrm{J}$ and Haier J: Organ-specific metastatic tumor cell adhesion and extravasation of colon carcinoma cells with different metastatic potential. Am J Pathol 169(3): 1064-1073, 2006.

31 Sleemana JP, Christofori G, Fodded R, Collarde JG, Berx G, Decraeneh $\mathrm{C}$ and Rüegg C: Concepts of metastasis in flux: The stromal progression model. Semin Cancer Biol 22: 174-186, 2012.

32 Wang D, Sun H, Wei J, Cen B and DuBois RN: CXCL1 is critical for pre-metastatic niche formation and metastasis in colorectal cancer. Cancer Res, 2017. doi: 10.1158/0008-5472.CAN-16-3199. [Epub ahead of print]

33 Roder D, Karapetis CS, Wattchow D, Moore J, Singhal N, Joshi R, Keefe D, Fusco K, Buranyi-Trevarton D, Sharplin G and Price TJ: Metastatic colorectal cancer treatment and survival: the experience of major public hospitals in South Australia over three decades. Asian Pac J Cancer Prev 16(14): 5923-5931, 2015.

34 Lazennec $\mathrm{G}$ and Richmond A: Chemokines and chemokine receptors: new insights into cancer-related inflammation. Trends Mol Med 16(3): 133-144, 2010.

35 Acharyya S, Oskarsson T, Vanharanta S, Malladi S, Kim J, Morris PG, Manova Todorova K, Leversha M, Hogg N, Seshan VE, Norton L, Brogi E and Massagué J: A CXCL1 paracrine network links cancer chemoresistance and metastasis. Cell 150(1): 165-178, 2012.

36 Verbeke H, Struyf S, Laureys G and Van Damme J: The expression and role of CXC chemokines in colorectal cancer. Cytokine Growth Factor Rev 22(5-6): 345-358, 2011.

37 Wang E, Zaman N, Mcgee S, Milanese JS, Masoudi-Nejad A and O'Connor-McCourt M: Predictive genomics: a cancer hallmark network framework for predicting tumor clinical phenotypes using genome sequencing data. Semin Cancer Biol 30: 4-12, 2015.

Received June 9, 2017

Revised June 30, 2017 Accepted July 6, 2017 